regetable tar, but it differs very materially from the latter in smell, for its odour is extremely disagreeable. It is soluble in alcohol, and turns of a thick brownish white colour when mixed well with water. By evaporating the spirit, the tar again floats on the surface. When igrited it burns like common tar, leaving a smell not unlike that of burnt feathers. The quantity obtained being too small for redistillation, $I$ was obliged to heat a little in a bulb glass to the temperature of ebullition, when a spirit was given off which took fire when bronght in contact with a lighted taper.

1 hope that my experiments will be repeated, provided the result is yet otherwise unknown; not that 1 think it of great consequence, but we must always feel interested in seeing the secrets of nature unfolded. Transmitting this account for insertion in your excellent journal, I remain, Sir, your obedient humble servant,

Southampton, Feb. 16th.

Henry Osborn, Chemist.

\section{BROWN BREAD.}

\section{To the Editor of ThF LanceT.}

Sin,-Allow me, through the medium of th your valuable journal, to call the attention of the profession to the uses and effects of brown bread, both as a remedy in habitual costiveness, and also as an article of diet. I am induced to make these observations, in consequence of having myself experienced both its value as a remedy in the abovementioned state of the bowels, and its injurious effects when used for any length of time as an article of diet. 1ts medicinal virtues are really surprising. It scarcely ever fails to remove, in a very short time, that habitual costiveness which so generally accompanies a sedentary life. But when its use is continued for some time after the costive state of the bowels has been removed, its effects are most injurious. It then produces all the symptoms of dyspepsia, and irritation of the whole alimentary canal. The laxative effect arises from the particles of bran which it contains, acting as a mechanical stimulant, and increasing the peristaltic action of the intestines. Now the very manner of its action as a laxative, proves its unitness as an article of diet; as it is necessary for all substances, in order that they may have any mechanical effect upon the bowels, to possess the power of resisting the action of the gastric juice, and all substances that have this power (for instance the rind of vegetables, under which denomination bran comes) also pos. sess the power of producirg great derange. ment of the alimentary canal, and I believe
I have, in some cases, seen inflammation of the mucous membrane prodnced by the continued use of bread containing bran. Brown bread is very extensively usel in this city, aud is sold under the name of "Constitutional bread," a name of which it is not deserving. I will at some future period furnish you with cases illustrative of its effects. Believe me, Sir, your obedient scrvant,

\section{Harcourt-street, Dublin,}

Charles Wright. Feb. 13, 1835.

\section{S Y P H I L IS.}

"A gentleman who had been cured of a chancre at a distance from home, called to consult me whether he might consider himself as perfectly free from the disease. Wbilst he was taking great pains to explain to me how he had been salivated, and how long he had continued the use of mercury after the chancre was healed, I interrupted him by observing, that if he had continued the use of mercury till now, I could not pretend to say whether he was free from the discase. 'How then,' said the gentleman, 'am I to ascertain my real situation?' 'If', replied I, 'you find no symptoms in the couise of three months, the probability is that you will remain well till you expose yourself to a new source of infection.' In about six weeks he returnel, with a sore throat and copper spots. I explained to him that he should not blame his surgeon, who, even if he had known what was to happen, could not have prevented it. The patient went through a necessary course of mercury, till he was cured of every symptom; and then demanded, with some impatience, whether he was secure. "You are secure,' replied I, 'fr'om every return on the genitals, and on your skin and throat; but, as it is impossible for me to know whether your bones are contaminated, I cannot pretend to say whether you will have nodes in a few weeks' time.' He now began to comprehend the doctrine, and submitted to await the result. In about six weeks he actually had nodes; after the cure of which, by a serere salivation, I made no scruple to assure him, he was perfectly free from the disease."-Mr. Krunter's last conversation with Dr. Adams.

The effects of the venereal poison upon the human body, Mr. Hunter describes under the three different states of "contamination," "disposition," and "action." By contamination, he implied the entrance of the poison into, and its contact with, some part of the body. By disposition, the intermediate state between contamination and the breaking out of the disease, or the state of action. 
First, of the state of contamination.

Mr. Hunter wes of opinion that mercury, if given immediately before, or upon, the appearance of cbancre, prevents cortamination. He says, "If the poison were still capable of circulating after its visible effects were cured, mere mercury given in the time of a chancre can be of little service, as it can only assist $i_{i}$ the cure of a chancre, but cannot preserve the constitution, which does not agree with experience; for practice informs us, that not one in fifty would escape the lues venerea, if the chancre were only cured tocally; - so that mercury has the power of preventing a disposition from forming, and therefore is necessary to be given, while we suppose absorption going on, or while there is matter that nay be absorbed." -Page 429, secund edition, by Dr. Adams.

Dr. Adams, commenting on this opinion, observes, - "This is a fair inference, but its truth can never be ascertained, because the primary diseased action, whether chancre or gonorrhoa, has always commenced before mercury is applied."-Page 433.

The experience of the military surgeons, especially of $\mathrm{Mr}$. Guthrie and $\mathrm{Mr}$. Rose, although of no very recent date, throws considerable light on this important point. $\mathrm{Mr}$. Guthrie, in an interesting paper, in the Medico-Chirurgical Transactions, I believe, vol. viii, observes, - "During the last eighteen months, in the York Hospital, Chelsea, Mr. Dease, Dr. Arthur, Dr. Gordon, and inyse'f, have been in the habit of treating all cases of ulcers on the penis, whatever form or appearance they might have, by simple mild means; that is, by dry lint, or ointment, or lotions, for the most part not containing mercury, in order to obviate the oljections that might be made to the application of mercury in any form; and of near one hundred cases which have been treated in this manner, all the ulcers healed without the use of mercury. The primary sores were of every description, from the superficial ulcer of the prepuce and glans, to the raised ulcer of the prepuce, the excavated ulcer of the glans, and the irritable and sloughing ulcer of those parts.

"The secondary symptoms, in the cases alluded to, amounting to one-tenth of the whole, and which were treated on the antiphlogistic plan, have hitherto been nearly confined to the first order of parts - that is, the bones have in two cases only been attacked, and they have equally been cured without mercury."

"A gentleman, aged 27, contracted venereal sores in the month of June, 1833, and was put under a mercurial course, during which time the sores healed, but he still continued the mercury. While under the influence of that medicine, he indulged in impure connection, the consequence of which was a fresh crop of vexererl sores, three or four. The mercurial corrse was

No. 651 . prolonged until the healing of these second sores. About two montbs after this, secondary symptoms made their appearance, for which he was again salivated, with apparent success; but now a new enemy appeared in the field, in the shape of rheumatism, and he again applied for medical advice. The surgeon whom he consulted considering it a case of venereal rheumatism, arising from a portion of the poison still lurking in the system, advised a fourth mercurial course, but although attended with partial relief during the existence of the ptyalism, the rheumatism shortly after returned with re-' doubled violence, in which state the patient. came under my observation."

The observation of Mr. Hunter, "that. not one in fifty cases of chancre would es. cape the lues venerea, if cured locally," when placed in opposition with the experience of the military surgeons above stated, appears to prove one of two things, either that Mr. Hunter was mistaken, or that the poison has become much milder in its effects.

First-Was Mr. Hunter mistaken? Speaking of the acrimony of the poison, he observes, "Venereal matter must in all cases be the same; one quantity of matter cannot have a greater degree of poisonous quality than another; and if there be any difference, it is only in being more or less diluted, which produces no difference in its effects. One can however conceive, that it may be so far diluted as not to have the power of irritation. The variation of the symptoms in different persons depends upon the constitution and habit of the patient at the time. What happens in the inoculation of small-pox strengthens this opinion. Let the symptoms of the patient, from whom the matter is taken be good or bad, - let it be from one who has had many pustules, or from one who has had but few, let it be from the confluent or the distinct kind, applied in a large quantity or a small one, it produces always the same effect.

In a small pamphlet submitted to your notice, $I$ have stated my conviction, that the poison or poisons producing gonorrhoea and chancre do vary in their acrimony, and are arising de novo daily, - that the majority of venereal sores are not true syphilis, or, in other words, are not followed by secondary symptoms, and, therefore, require for their cure little or no mercury.

Now, if we can throw off the idea of the venereal poison being like small-pox, a specific, uniform, and unv rying poison, and believe that it varies in acrimony, not only from the constitution, but from the habits of life of the patient, then nothing is more reasonable than the belief that the effect will also vary in severity.

It is well known that the disease is milder in France than in this country, which would appear in a great measure to be owing to $3 \mathrm{H}$ 
the necessity on the part of the females for cleanliness and personal ablution, in order that they may not be placed out of the " pale of health" by medical superintend. ence.

Mr. Hunter's comparison of the venereal poison with small-pox is, I think, inadmissible; since, while small-pox produces $\mathrm{m}$ mallpox or nothing, three persons, having connection with one female, may have gonorrhoea, chancre, or bubo; and even these varieties may vary in acrimony. Now, while the cause which produces these diseases is one and the same, when once removed from the woman, it does not necessarily maintain its original character; the effect is not necessarily the same as the cause ; for, if so, the excoriation from venereal gonorrhcea must be chancre, and the excoriation of a chancre must be chancre also, or, if the discharge of the latter comes in contact with the mucous lining of the urethra, it should produce gonorrhœe, whereas, either of these effects is, as far as I have seen, extremely rare. While, therefore, it is possible Mr. Hunter might overrate the comparative number of cases, which, in the absence of mercury, would be followed by lues venerea, I hold it to be extremely probable that, at the time he wrote (now about fifty years since), the venereal disease or poison possessed a higher degree of acrimony.

At page 274 of Mr. Abernethy's Lectures on Surgery, speaking of the venereal dis. case as described by Hunter, he observes, "This disease has almost become extinct, or is so much modified as to be unlike that which Mr. Hunter has described, and which $I$ had an opportunity of observing in the earlier part of my life."

It is an opinion very generally entertained that venereal bubo is a sign of the absorp. tion of the poison, or, in other words, of the contamination of the system; there is, however, I believe, little reliance to be placed on this symptom, for, in the first place, any irritation in the urethra or on the penis may produce a bubo; in the next, secondary symptoms frequently follow a sore without bubo : in the third place, bubo, or inflammation of the glands in the groin, or indeed of any part of the body, frequently arises from cold or rheumatism, or any like cornmon causes. While such explanations are at hand, why have recourse to hypothesis, for after all that has been said and done, I contend, that the opinion that the venereal poison is absorbed into the body, is not substantiated by facts or warranted by symptoms.

I shall here take leave, I trust not uncourteously, to express, freely and unequivocally, my dissent from the views and treatment of bubo as inculcated at page 205 of Mr. Judd's recent work on Urethritis and Syphilis. Unless it can be proved that secondary symptoms have arisen from bubo, unaccompanied by any primary sore, I am quite at a loss to comprehend the propriety of treating it as a venereal disease. Unless it can be proved in the language of Hunter, " that bubo is a venereal alsscess," exactly similar in its nature and effects to a chancre, the only difference being in size; unless all this is established by facts (with the opposing evidence , it is fair to treat the opinion as hypothetical.

For 1st. I have inoculated a sound person three separate times from three distinct buboes (following venereal sores), without in either case producing contamination.

2ndly. "With respect to absorption, even when the disease is followed by secondary symptoms." Mr. Hunter says, "we may observe, that even the blood of a pocky person has no power of contaminating, and is not capable of giving the aisease to another, even by inoculation; for if it were capable of irritating a sound sore to a venereal inflammation, no person that has this matter circulating, or has the lues venerea, could escape having a venereal sore whenever he is bled, or receives a scratch with a pin. the part so wounded turning into a chancre. For if the venereal matter be on the point of the lancet or on the point of the pin, the punctures must become chancres."

Again, as to the venereal bubo containing chancrous matter, it might as well be argued, that when suppuration takes place in any part of the glandular system during variola, scarlatina, and rubeola, such matter would possess the specific property of each disease; or, to go on with the argument, that the matter formed from suppuration of a gland, from a dissecting wound, must necessarily contain matter of the same quality as the original. The same reasoning, for aught I see, might be applied to natural poisons, the poison of the viper, \&c. The treatment of bubothen, in my humble opinion (in the language of $\mathrm{Dr}$. Titley), "is not to be influenced by the consideration, fousded on sheer hypothesis, that the glandular enlargernent depends upon the absorp. tion of a specific virus."-Page 119.

\section{2ndly. Of the state of disposition.}

Mercury cures the action but not the disposition. "Hence when a part is contami. nated, and under the disposition to disease which should show itself at a certain time, mercury will protract that period, and the disease will not show itself so long as the constitution is under the influence of morcury. But all this time the disease will not be cured. After the mercurial irritation has ceased, the venereal disposition, which has existed ever since the parts were contaminated, will come into action; that is, the disease will appear, and in this state will be cured. These principles, being established, explain at once the difficulty that 
existed, whilst attempts were made to aradicate the virus." Page 433 .

$3 r d l y$. Of the state of action.

"Mercury cures the venereal action." Until we define more precisely what is venereal action, it is impossible to speak definitely of the action of mercury, sinco daily experience convinces me, that many effects imputed to the venereal poison, are neither more nor less than productions of the mercury. I conclude then,

1st. That the occurrence of vencreal sores while the body is under the influence of mercury, proves that mercury does not prevent contamination.

2ndly. That mercury does not prevent the disease from runnixg a certain course. By this I mean the occurrence of lues venerea. If this be true, it follows,

3 rdly. That the continuance of the mercury for three weeks after the primary sores have healed, with a view to eradicate the virus, is perfectly unjustifiable both in theory and practice.

4 thly. That there is no legitimate ground for believing that the venereal poison is ever absorbed in to the body.

5thly. That the majority of venereal sores are not followed by lues venerea.

6 thly. That the venereal poison varies in acrimony.

7 thly. That the majority of venercal sores, which are not followel by lues venerea, are, in all probability, the production of the sume poison that produces true chancre, only in a minor degrce of acrimony.

It was my intention to have stated the facts on which I found the opinion, that the disease called "venereal rheumatism" is, more properly speaking, mercurial, - a consequence of the remedy and not of the disease. As, however, these remarks have extended to a length beyond my original intention, I cannot presume to trespass farther at present on your valuable space.

29, Poultry, Feb. 9, 1836.

Francis Eagle.

NEW RESEARCHES ON THE

\section{MOVEMENTS OF THE HEART.}

Tak theory of the movements and bruits of the heart still remains in great obscurity, notwithstanding the labours of Messrs. Boulllaud, Pigeaux, Marc D'Esprane, Rovant, and MAGENoIE, each of whom has formed a different theory. The researches of the Dublin Commission, composed of Drg. Corrigin, Law, Nolan, \&c., ought to give birth, if not to the best, at least to the newest explauation, which is always something in the value of an hypothesis. In the mean time, Mons. BEsu, one of the most intelligent internes of the school of Paris, after having made several experiments on cold and warm blooded animals, has arrived at conclusions, that seem to be supported by the facts which he produces, however contradictory some of them may be to our received notions. We cannot detail the experimerts which form the basis of this long memoir, but will refer to them in such a manner as will render our description of M. BEAU's ideas intelligible.

The heart is the seat of two principal movements, which alternate with each other, and on whose existence all writers are agreed; in one, the inferior part of the heart or its apex is carried forwards; in the other it is the superior part or base of the heart which advances, while the point retires towards the dursal parietes of the chest; we may call the sound which attends the first of these movements the inferior sound (the first or dull bruit of authors); the second gives the superior bruit (the second, or clear sound). It is also very generally admitted that the first is a movement of contraction (systole); the second, a movement of dilatation. Laying aside the manner in which other authors have endeavoured to explain the mechanism of these two movements, and particularly of that by which the point of the heart is carried forwards against the parietes of the chest, let us see how M. BEAU renders an account of the latter phenomenon. In physiology, all reasoning should be founded on observation and experiment. This grand truth, first established by our countryman Bacon, for the natural sciences, is at the present day applied with success to the investigation of the laws that govern medicine and physiology, by two dist ${ }^{*}$ guished physicians, Messrs. Louis and Magendie: the necessity of abandoning bypothesis for observation is daily gaining more ground, and the time is doubtless not far off when the science of life will progress in proportion as we abandon idle speculation for the study of the phenomena of living bodies.

The first experiments made by $M . B E A u$ were performed on frogs. On exposing the heart of one of those animals we are struck with a double movement of dilatation. which is very remarkable, and affect at $3 \mathrm{H} 2$ 